\title{
Editorial
}

\section{Gram-Negative, Hospital-Acquired Infections: A Growing Problem}

\author{
Lennox K. Archibald, MD, FRCP, DTM\&H
}

This issue of Infection Control and Hospital Epidemiology contains multiple articles focusing on the clinical and public health implications of infections by extended-spectrum beta-lactamase (ESBL)-producing Enterobacteriaceae, Pseudomonas aeruginosa, and Acinetobacter baumannii and their resistance profiles to commonly prescribed antimicrobials. The first five articles in this issue $\mathrm{e}^{1-5}$ add to a growing body of literature that substantiates the following: (1) as in North America, gramnegative pathogens are important causes of hospitalacquired infections in Europe and Asia; (2) antimicrobial resistance among gram-negative pathogens is a global problem; (3) the pathogens encountered in healthcare institutions in North America, Europe, and Asia are, for the most part, similar; (4) the risk factors (eg, device use or antimicrobial overprescribing) associated with hospitalacquired infections and antimicrobial resistance are universal; (5) surveillance activities are important for the characterization and control of gram-negative, healthcareassociated infections in Europe and Asia and require appropriate integration of epidemiologic, microbiologic, and clinical data; and (6) the microbiology laboratory is important in the characterization and control of healthcare-associated infections and antimicrobial resistance.

In the first article in this issue of Infection Control and Hospital Epidemiology, Yu et al. used a combination of phenotypic and molecular typing methods to demonstrate clonal dissemination of ESBL-producing Klebsiella pneumoniae in 24 hospitals located in several regions of Taiwan. ${ }^{1}$ Using a combination of ribotyping and pulsed-field gel electrophoresis (PFGE), they identified two epidemic clones in hospitals located in the northern and central regions of the country. Within each clone, however, there were variations in the antibiogram and isoelectric focusing profiles of isolates. Their findings suggest significant intrahospital and interhospital clonal transmission of ESBL-producing $K$. pneumoniae and confirm the ubiquity of this resistant pathogen in major teaching hospitals in all regions of Taiwan. They provide evidence that interinstitutional transmission of ESBL-producing $K$. pneumoniae parallels the movement of mobile patient populations. Previously, Monnet et al. used molecular typing to demonstrate interhospital transmission of ESBL-producing Klebsiella species in one region of the United States, and concluded that physical proximity of hospitals might facilitate interhospital transmission of ESBL-producing $K$. pneumoniae. ${ }^{6}$ More recently, the results of a case-control study at a district teaching hospital in Taiwan identified ceftazidime use as an independent risk factor associated with acquisition of ESBL-producing K. pneumoniae. ${ }^{7}$ The findings of Yu et al. are consistent with those of a 2004 report of nationwide surveillance data on antimicrobial resistance among gram-negative pathogens in 21 Taiwanese medical centers-gramnegative bacterial resistance is prevalent in medical centers and regional hospitals throughout Taiwan. ${ }^{8}$

In the second article in this issue of Infection Control and Hospital Epidemiology, Rodriguez-Baño et al. report the results of a multicenter study conducted to characterize the clinical features and molecular epidemiology of $A$. baumannii infections in 28 hospitals in Spain that together served a catchment area containing approximately $25 \%$ of the Spanish population. ${ }^{2}$ During November 2000, every new patient with $A$. baumannii infection or colonization was included in the study: all isolates were typed first using phenotypic methods and then using genotyping with PFGE. Twenty-five (89\%) of the 28 participating hospitals 
identified 206 patients with $A$. baumannii infection or colonization- $53 \%$ of the patients were infected and the rest were colonized. Affected patients were most often in intensive care units (ICUs) (47\%), followed by the medical and surgical wards (25\% in each). The highest $A$. baumannii rates were found in paraplegic centers. The most frequently affected body site was the respiratory tract, followed by surgical wounds and the urinary tract. Most isolates from patients in the paraplegic center came from the urinary tract, suggesting that the urine of catheterized patients may be an important reservoir. Rodríguez-Baño et al. found no association between $A$. baumannii infection or colonization and geographic location, hospital size, type of hospital, or service specialty. Although a predominant clone was observed in 14 of the 28 hospitals and imipenem resistance was more frequent $(57 \%)$ in university hospitals, wide heterogeneity was found among isolates of $A$. baumannii from different hospitals. This study confirmed that $A$. baumannii was present in most of the hospitals that provide services to nearly $25 \%$ of the population of Spain and that the respiratory tract was the most common site of infection. Of particular concern were the difficulty in distinguishing clinically significant infection from colonization and the unnecessary antimicrobial use that might be associated with the latter.

In the third article in this issue of Infection Control and Hospital Epidemiology, Ortega et al. describe a singlecenter, retrospective cohort study characterizing the epidemiology and outcomes associated with multidrugresistant $P$. aeruginosa infection and colonization among critically ill patients in the ICU of a university hospital in the Netherlands. ${ }^{3}$ Their study integrated comprehensive clinical, epidemiologic, and microbiologic data components. To ascertain the mode of transmission among patients, strains of multidrug-resistant isolates were genotyped by amplified fragment-length polymorphism. Multidrug resistance was defined as $P$ aeruginosa resistant to at least two of the following: aminoglycosides, ceftazidime, ciprofloxacin, imipenem, and piperacillin. Patients found to be colonized or infected with multidrug-resistant $P$. aeruginosa were managed using barrier precautions and personnel who had direct contact with these patients wore gowns and gloves. Eighteen (34\%) of the 53 isolates from patients who harbored $P$. aeruginos $a$ were multidrug resistant; most (55\%) of these resistant isolates were associated with ventilatorassociated pneumonia. On logistic regression analysis, independent risk factors for multidrug-resistant $P$. aeruginos $a$ included maxillary sinus origin of the pathogen, prolonged duration of ICU stay, mechanical ventilation, a long duration of ceftriaxone therapy, and a high lung injury score that was based on oxygenation and ventilation parameters. Molecular typing suggested that $64 \%$ of the multidrug-resistant strains were likely transmitted via cross-colonization and $36 \%$ had probably originated endogenously. Surprisingly, Ortega et al. did not observe any differences in mortality rates between patient groups who harbored multidrug-resistant isolates and those who did not.
In the fourth article in this issue of Infection Control and Hospital Epidemiology, Lee et al. provide a snapshot of the clinical and epidemiologic correlates of ESBL-producing $K$. pneumoniae in a teaching hospital in Korea. ${ }^{4}$ Korean authorities have claimed that antimicrobial-resistant bacteria are more prevalent in Korea than in other industrialized countries. ${ }^{9}$ One publication estimated that $23 \%$ of Korean $K$. pneumoniae isolates produced ESBLs. ${ }^{10}$ More recent studies have suggested that $39 \%$ of $K$. pneumoniae and Escherichia coli strains obtained from various regions in Korea produced ESBLs; $27 \%$ of $K$. pneumoniae bloodstream isolates were ESBL producing. ${ }^{11,12}$ In an effort to identify measures to control the acquisition of ESBL-producing $K$. pneumoniae among patients in a neurosurgical ICU, Lee et al. conducted a nested case-control study to identify independent risk factors amenable to modification. Among 29 respiratory ESBLproducing $K$. pneumoniae isolates identified by phenotypic methods only, there was one cluster of 6 isolates during a 2-week period with the same antibiogram, but 17 other antibiograms among the other 23 isolates; there was no more clustering suggesting spread of particular strains. On multivariate analysis, exposure to third-generation cephalosporins was the only independent risk factor associated with ESBL-producing $K$. pneumoniae that was amenable to modification. In looking back to ascertain the reasons for exposure to third-generation cephalosporins, Lee et al. found that $63 \%$ of the 51 study patients (ie, 24 casepatients and 27 control-patients) exposed to third-generation cephalosporins had been treated inappropriately. Focusing solely on the control of third-generation cephalosporins in the unit, they demonstrated a statistically significant decrease in the frequency of ESBL-producing $K$. pneumoni$a e$ in the respiratory tracts of patients admitted to the neurosurgical ICU.

The study by Thouverez et al. ${ }^{5}$ in this issue of Infection Control and Hospital Epidemiology sought to evaluate the utility of screening cultures in the control of ESBL-producing Enterobacteriaceae. They looked retrospectively at the results of screening and clinical cultures from patients admitted to two ICUs in a university-affiliated hospital in France during a 4-year period. Patients whose clinical or screening cultures yielded ESBL-producing Enterobacteriaceae were placed in barrier precautions with hospital personnel using gowns and gloves as part of contact precautions for all colonized or infected patients and their environment. Using dates of inpatient stay, ICU location, isolation of ESBL-producing Enterobacteriaceae, and PFGE profiles for patients, they defined clusters of cases ostensibly due to transmission between patients. Thouverez et al. found that Enterobacter aerogenes was the predominant ESBL-producing Enterobacteriaceae, and documented a significant decrease in the number of colonized or infected patients following the implementation of screening cultures to detect colonized patients and contact precautions for colonized patients, without making any modifications in antimicrobial use or prescribing practices during the study period. These findings are similar to other recently published data showing that ESBL-produc- 
ing $E$. aerogenes have been the main source of ESBL dissemination in other regions in France. ${ }^{13-15}$ Bertrand et al. also emphasized the importance of control through early detection of colonization with ESBL-producing organisms in high-risk areas (ie, ICUs), and contact precautions for colonized patients. ${ }^{14}$

After the outbreak had been controlled, Thouverez et al. observed that using the results of clinical specimen cultures alone to select patients for isolation and barrier precautions was probably sufficient to control ESBL-producing Enterobacteriaceae. In this setting with a low prevalence of ESBL-producing Enterobacteriaceae, screening cultures were neither sensitive nor cost-effective (screening cultures failed to detect $50 \%$ of the probable imported cases on admission). Thouverez et al. noted that they had stopped screening for ESBL-producing Enterobacteriaceae, which will allow them to test their hypothesis that clinical specimen cultures are sufficient to maintain control in a non-outbreak setting.

As mentioned above, the first five articles in this issue of Infection Control and Hospital Epidemiology make it clear that infections due to ESBL-producing Enterobacteriaceae are causing important problems for ICU patients around the globe, that heavy use of particular antimicrobials creates a selective advantage for these strains, and that transmission of resistant strains from patient to patient often is involved. A recent multicenter study involving 12 hospitals on 6 continents concluded that ESBL-producing $K$. pneumoniae were 9-fold more likely to be the cause of nosocomial than community-acquired $K$. pneumoniae bacteremia and that 7 of 10 hospitals with multiple ESBL-producing isolates had multiple isolates with the same genotypic pattern, suggesting nosocomial transmission. Moreover, 6 of these 7 hospitals had not been using contact isolation for patients with ESBL-producing strains. ${ }^{16}$ The authors concluded that appropriate infection control and antibiotic management strategies were needed to stop the spread of this emerging form of resistance.

\section{REFERENCES}

1. Yu W-L, Winokur PL, Jones RN, Sader HS. Surveillance in Taiwan using molecular epidemiology for extended-spectrum beta-lactamase-produc- ing Klebsiella pneumoniae. Infect Control Hosp Epidemiol 2004;25:812818.

2. Rodriguez-Baño J, Cisneros JM, Fernández-Cuenca F, et al. Clinical features and epidemiology of Acinetobacter baumannii colonization and infection in Spanish hospitals. Infect Control Hosp Epidemiol 2004;25:819824.

3. Ortega B, Groeneveld ABJ, Schultsz C. Endemic multidrug-resistant Pseudomonas aeruginosa in critically ill patients. Infect Control Hosp Epidemiol 2004;25:825-831.

4. Lee S-O, Lee ES, Park SY, Kim SY, Seo Y-H, Cho YK. Reduced use of third-generation cephalosporins decreases the acquisition of extendedspectrum beta-lactamase-producing Klebsiella pneumoniae. Infect Control Hosp Epidemiol 2004;25:832-837.

5. Thouverez M, Talon D, Bertrand X. Control of Enterobacteriaceae producing extended-spectrum beta-lactamase in intensive care units: rectal screening may not be needed in non-epidemic situations. Infect Control Hosp Epidemiol 2004;25:838-841.

6. Monnet DL, Biddle JW, Edwards JR, et al. Evidence of interhospital transmission of extended-spectrum beta-lactam-resistant Klebsiella pneumoniae in the United States, 1986 to 1993. Infect Control Hosp Epidemiol 1997;18:492-498.

7. Lin MF, Huang ML, Lai SH. Risk factors in the acquisition of extended-spectrum beta-lactamase Klebsiella pneumoniae: a case-control study in a district teaching hospital in Taiwan. I Hosp Infect 2003:53:39-45.

8. Lauderdale TL, McDonald LC, Shiau YR, et al. The status of antimicrobial resistance in Taiwan among gram-negative pathogens: the Taiwan Surveillance of Antimicrobial Resistance (TSAR) program, 2000. Diagn Microbiol Infect Dis 2004;48:211-219.

9. Chong $Y$, Lee K. Present situation of antimicrobial resistance in Korea. Infect Chemother 2000;6:189-195.

10. Paj $\mathrm{H}$. The characteristics of extended-spectrum beta-lactamases in Korean isolates of Enterobacteriaceae. Yonsei Med J 1998;39:514519.

11. Kim BN, Woo JH, Kim MN, Ryu J, Kim YS. Clinical implications of extended-spectrum beta-lactamase-producing Klebsiella pneumoniae bacteraemia. J Hosp Infect 2002;52:99-106.

12. Jeong SH, Bae IK, Kwon SB, et al. Investigation of extended-spectrum beta-lactamase produced by clinical isolates of Klebsiella pneumoniae and Escherichia coli in Korea. Lett Appl Microbiol 2004;39:4147.

13. Eveillard M, Biendo M, Canarelli B, et al. Spread of Enterobacteriaceae producing broad-spectrum beta-lactamase and the development of their incidence over a 16-month period in a university hospital center. Pathol Biol 2001;49:515-521.

14. Bertrand X, Hocquet D, Boisson K, Siebor E, Plesiat P, Talon D. Molecular epidemiology of Enterobacteriaceae producing extendedspectrum beta-lactamase in a French university-affiliated hospital. Int J Antimicrob Agents 2003;22:128-133.

15. Arpin C, Dubois V, Coulange L, et al, Extended spectrum betalactamase-producing Enterobacteriaceae in community and private health care centers. Antimicrob Agents Chemother 2003;47:35063514.

16. Paterson DL, Ko WC, Von Gottberg A, et al. International prospective study of Klebsiella pneumoniae bacteremia: implications of extendedspectrum beta-lactamase production in nosocomial infections. Ann Intern Med 2004:140:26-32. 